\title{
Towards generalized measures grasping CA dynamics
}

\author{
Jan M. Baetens ${ }^{\star}$ and Bernard De Baets \\ KERMIT, Department of Applied Mathematics, Biometrics and Process Control, \\ Ghent University, Coupure links 653, Gent, Belgium \\ $\{j$ an. baetens, bernard.de.baets\}@ugent.be
}

\begin{abstract}
In this paper we conceive Lyapunov exponents, measuring the rate of separation between two initially close configurations, and Jacobians, expressing the sensitivity of a CA's transition function to its inputs, for cellular automata (CA) based upon irregular tessellations of the $n$-dimensional Euclidean space. Further, we establish a relationship between both that enables us to derive a mean-field approximation of the upper bound of an irregular CA's maximum Lyapunov exponent. The soundness and usability of these measures is illustrated for a family of 2-state irregular totalistic CA.
\end{abstract}

Key words: irregular tessellation, Jacobian, Lyapunov exponent

\section{Introduction}

Since their conceptualization by von Neumann [31] more than 60 years ago, cellular automata (CA) have proven their usefulness in applied sciences as adequate modeling tools in numerous scientific fields, such as epidemiology [21,33], demography $[7,8,15]$, microbiology [23, 24], traffic engineering [9], hydrology and geology $[10,12,13,22,29]$, and numerous others $[1,18,20,25]$, while in exact sciences much attention has been given to the complex spatio-temporal dynamics of these intrinsically simple discrete dynamical systems [6, 26,35-37]. In contrast to continuous dynamical systems such as ordinary and partial differential equations (ODE and PDE) that often allow to investigate the system's stability properties without having to solve the ODE or PDE, adequate conclusions about a CA's dynamical properties can mostly only be drawn from extensive computer simulations [17,35,37], except for the class of additive CA [34]. This has motivated several researchers to develop quantitative measures for discriminating between the behavioral classes of CA distinguished by Wolfram [35], such as the Hamming distance [6,35], the Langton parameter [19], Lyapunov exponents [11,27, 28], entropies and dimensions [16], and others [38], or by relying on mean-field approximations [14].

Among these measures, Lyapunov exponents that were first introduced in 1D CA as the propagation speed of the damage front originating from an initial

\footnotetext{
* Corresponding author
} 
perturbation of the state of one of the cells [36], and later described rigorously for 1D CA [26], are perhaps the most promising as indicated by their prevalent use in papers on the phenomenology of CA $[4-6,11,26-28]$. Besides, their manifold use for the characterization of continuous dynamical systems makes them easily accessible for researchers that are not acquainted with the typicalities of CA. Most frequently, Lyapunov exponents have been applied to characterize the dynamics of $1 \mathrm{D} \mathrm{CA}$, since in this case, the damage front can propagate only to the left or to the right of the initially perturbed cell, enabling a sound formulation of right and left Lyapunov exponents [26]. Clearly, if higher-dimensional CA are at stake, the usefulness of such directional Lyapunov exponents is strongly hampered since the damage front in, for instance 2D CA, can propagate circularly from an initial perturbation. For that reason, Bagnoli et al. [6] formulated a non-directional Lyapunov exponent, which has been applied in combination with a measure expressing the sensitivity of a CA's transition function to its inputs and based upon Boolean derivatives [30], to study 1D CA, as well as 2D lattice gas automata $[4,5]$. However, the latter measure is formulated in such a way that its use is limited to CA based upon regular tessellations of $\mathbb{R}^{n}$ since it assumes that the neighborhood structure is fixed, which is clearly not the case if irregular tessellations are at stake. Hence, in order to grasp the dynamics of CA based upon irregular tessellations, described rigorously in [2], as well as to compare the dynamics of CA defined upon different tessellations of $\mathbb{R}^{n}$, a generalized definition of this measure should be formulated, and its relationship with the non-directional Lyapunov exponents should be readdressed.

In Section 2 we outline the mathematical preliminaries that are necessary for a proper understanding of Section 3 in which we conceive Lyapunov exponents and Jacobians for irregular CA, and establish a measure grasping the CA's sensitivity to its input by relying on the latter. An exemplary simulation study of 2-state irregular totalistic CA concludes this paper.

\section{Preliminaries}

We state the definition of a cellular automaton on an arbitrary tessellation of a $n$-dimensional Euclidean space, which constitutes an extension to the classical CA paradigm that predominantly relies on regular tessellations of $\mathbb{R}^{n}$ ever since von Neumann's pioneering work [32].

Definition 1. (Cellular automaton)

$A$ cellular automaton $(C A) \mathcal{C}$ can be represented as a sextuple

$$
\mathcal{C}=\left\langle\mathcal{T}, S, s, s_{0}, N, \Phi\right\rangle,
$$

where

(i) $\mathcal{T}$ is a countably infinite tessellation of a $n$-dimensional Euclidean space $\mathbb{R}^{n}$, consisting of cells $c_{i}, i \in \mathbb{N}$.

(ii) $S$ is a finite set of $k$ states, often $S \subset \mathbb{N}$. 
(iii) The output function $s: \mathcal{T} \times \mathbb{N} \rightarrow S$ yields the state value of cell $c_{i}$ at the $t$-th discrete time step, i.e. $s\left(c_{i}, t\right)$.

(iv) The function $s_{0}: \mathcal{T} \rightarrow S$ assigns to every cell $c_{i}$ an initial state, i.e. $s\left(c_{i}, 0\right)=s_{0}\left(c_{i}\right)$.

(v) The neighborhood function $N: \mathcal{T} \rightarrow \bigcup_{p=1}^{\infty} \mathcal{T}^{p}$ maps every cell $c_{i}$ to a finite sequence $N\left(c_{i}\right)=\left(c_{i_{j}}\right)_{j=1}^{\left|N\left(c_{i}\right)\right|}$, consisting of $\left|N\left(c_{i}\right)\right|$ distinct cells $c_{i_{j}}$.

(vi) $\Phi=\left(\phi_{i}\right)_{i \in \mathbb{N}}$ is a family of functions

$$
\phi_{i}: S^{\left|N\left(c_{i}\right)\right|} \rightarrow S,
$$

each $\phi_{i}$ governing the dynamics of cell $c_{i}$, i.e.

$$
s\left(c_{i}, t+1\right)=\phi_{i}\left(\tilde{s}\left(N\left(c_{i}\right), t\right)\right),
$$

where $\tilde{s}\left(N\left(c_{i}\right), t\right)=\left(s\left(c_{i_{j}}, t\right)\right)_{j=1}^{\left|N\left(c_{i}\right)\right|}$.

Although the complexity measures in the subsequent sections are derived for any CA that obeys the former definition, in the simulation study presented in the final section of this paper we focus on a family of totalistic CA, which we define a follows.

Definition 2. (Totalistic cellular automaton) A totalistic cellular automaton $(C A)$ is a $C A$ for which $S \subset \mathbb{N}$, and for which there exists a $\Omega: \mathbb{N} \rightarrow S$ such that

$$
s\left(c_{i}, t+1\right)=\phi_{i}\left(\tilde{s}\left(N\left(c_{i}\right), t\right)\right)=\Omega\left(\sigma_{i}\right),
$$

where $\sigma_{i}=\sum_{j=1}^{\left|N\left(c_{i}\right)\right|} s\left(c_{i_{j}}, t\right)$.

For the sake of uniformity, we also set up an enumeration scheme for irregular totalistic CA in accordance with the enumeration developed for their regular counterparts. Such a concise enumeration scheme allows to identify every $\phi_{i}$ that can be formulated, given the number of possible states $k$, by means of an unique number, commonly referred to as the rule number. To overcome the unboundedness of $\sigma_{i}$ that arises from allowing irregular tessellations of $\mathbb{R}^{n}$, we introduce an upper bound $\theta$ on $\sigma_{i}$ such that $\Omega\left(\sigma_{i}\right)=\Omega(\theta)$ if $\sigma_{i} \geq \theta$. As such, the rule number for a $k$-state, $\theta$-sum irregular totalistic CA, denoted $R_{\theta}^{\mathrm{T}}$, can then be found from its base- $k$ representation, containing $\mu=\theta+1$ digits, $z_{\theta} z_{\theta-1} \cdots z_{2} z_{1} z_{0}$ as

$$
R_{\theta}^{\mathrm{T}}=z_{\theta} k^{\mu-1}+z_{\theta-1} k^{\mu-2}+\ldots+z_{2} k^{\mu-(\mu-2)}+z_{1} k^{\mu-(\mu-1)}+z_{0},
$$

where $z_{f} \in\{0,1, \ldots, k-1\}$ represents the state value assigned to $c_{i}$ at the $t+1$-th time step if $\sigma_{i}=f$. A total of $k^{\theta+1}$ different rules can be enumerated for this family of irregular CA. 


\section{Lyapunov exponents and Jacobians for irregular CA}

\subsection{Lyapunov exponents}

Let $s_{0}$ and $s_{0}^{*}$ be two initial configurations of a 2-state CA for which $S=\{0,1\}$, such that there is only one $c_{i} \in \mathcal{T}$ for which $s_{0}\left(c_{i}\right) \neq s_{0}^{*}\left(c_{i}\right)$, i.e. $s_{0}^{*}$ constitutes the smallest possible perturbation of $s_{0}$. In what follows, we will refer to a cell $c_{i}$ for which $s\left(c_{i}, t\right) \neq s^{*}\left(c_{i}, t\right)$ as a defective or perturbed cell, or in short, as a defect. Further, if we define $\epsilon_{t}$ as the number of defective cells at the $t$-th time step, i.e.

$$
\epsilon_{t}=\left|\left\{i \mid s\left(c_{i}, t\right) \neq s^{*}\left(c_{i}, t\right)\right\}\right|,
$$

the quantity

$$
\lambda=\lim _{t \rightarrow \infty} \frac{1}{t} \log \left(\frac{\epsilon_{t}}{\epsilon_{0}}\right),
$$

can be intuitively interpreted as a maximum Lyapunov exponent (MLE). Yet, as indicated by Bagnoli et al. [6], we must take into account that a CA's discrete nature can cause defects to annihilate each other such that the quantity given by the right-hand side of Eq. (3) approaches zero as $t \rightarrow \infty$. Indeed, we observed this tendency for the family of irregular CA covered in this paper. As suggested by Bagnoli et al. [6], this artifact can be overcome by keeping track of the evolution of all the defects that arise during the CA's evolution, though they neglected to include an algorithmic procedure that allows a proper evaluation of $\epsilon_{t}$, and hence of $\lambda$. For that reason, we provide a brief algorithmic procedure that enables the calculation of the non-directional Lyapunov exponent of a CA (Algorithm 1).

It should be emphasized that, notwithstanding Eq. (3) demands to evaluate $\lambda$ as $t \rightarrow \infty$, practical considerations make us to calculate the MLE for finite $T$, and for finite tessellations $\mathcal{T}^{*}$ of a compact subset of $\mathbb{R}^{n}$. The MLE has been used before to classify both elementary and totalistic $1 \mathrm{D}$ cellular automata $[6$, 4], and is applied in our work to quantitatively describe the dynamics of 2-state, 5 -sum irregular totalistic CA.

\section{$3.2 \quad$ Jacobians}

In order to express the sensitivity of a CA's transition function $\phi_{i}$ to its input $\tilde{s}\left(N\left(c_{i}\right), t\right)$, we can construct a Jacobian matrix $J$ that has $\left|\mathcal{T}^{*}\right| \times\left|\mathcal{T}^{*}\right|$ entries:

$$
J_{i j}= \begin{cases}\frac{\partial s\left(c_{i}, t+1\right)}{\partial s\left(c_{j}, t\right)} & , \text { if } c_{j} \in N\left(c_{i}\right), \\ 0 & , \text { else }\end{cases}
$$

where $\frac{\partial s\left(c_{i}, t+1\right)}{\partial s\left(c_{j}, t\right)}$ is the Boolean derivative, introduced in CA by Vichniac [30]. If altering $s\left(c_{j}, t\right)$ affects $s\left(c_{i}, t+1\right)$, this Boolean derivative equals one, whereas it equals zero if such an alteration has no influence on the outcome of $\phi_{i}\left(\tilde{s}\left(N\left(c_{i}\right), t\right)\right)$. In contrast with the Jacobian of an elementary CA, the Jacobian of a CA based upon irregular tessellations of $\mathbb{R}^{n}$ is not tridiagonal. 


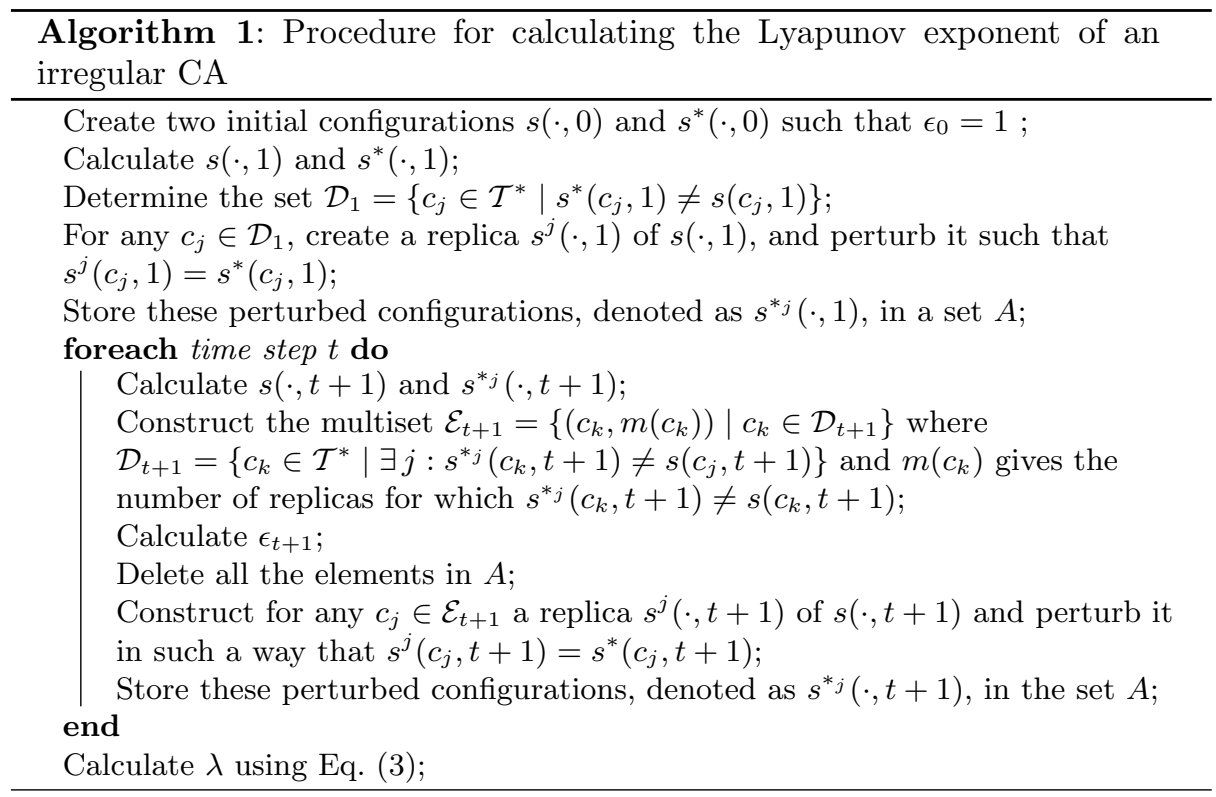

Considering the variability of $\left|N\left(c_{i}\right)\right|$ in irregular $\mathrm{CA}$, the average proportion of cells $c_{j}$ in $N\left(c_{i}\right)$ that affects $s\left(c_{i}, t+1\right)$ is given by

$$
\mu(t)=\frac{1}{\left|\mathcal{T}^{*}\right|} \sum_{c_{i}} \frac{1}{\left|N\left(c_{i}\right)\right|} \sum_{j=1}^{\left|N\left(c_{i}\right)\right|} J_{i i_{j}} .
$$

Essentially, $\mu(t)$ expresses the sensitivity of a CA's transition function to its inputs. Its geometric mean $\bar{\mu}$ after a large number of time steps $T$ is

$$
\bar{\mu}=\left(\prod_{t=1}^{T} \mu(t)\right)^{\frac{1}{T}} .
$$

Understandably, higher values of $\bar{\mu}$ indicate a higher sensitivity of $\phi_{i}$ to its input $\tilde{s}\left(N\left(c_{i}\right), t\right)$. Since the sensitivity is, within the outer summation of Eq. (5), normalized for every $c_{i}$ with respect to $\left|N\left(c_{i}\right)\right|$, it can be used to characterize a CA regardless of the tessellation it is based upon. Hence, it is an appropriate measure that can be exploited to compare the dynamics of CA that are based upon the same transition function $\phi_{i}$, but employ different tessellations of $\mathbb{R}^{n}$.

\subsection{Assessing an upper bound for Lyapunov exponents of irregular CA}

If we define the mean connectivity of $\mathcal{T}^{*}$ as

$$
\bar{V}=\frac{1}{\left|\mathcal{T}^{*}\right|} \sum_{c_{i}}\left|N\left(c_{i}\right)\right|,
$$


and we indicate that the right-hand side Eq. (3) measures the average rate of separation of two trajectories in phase space during one time step, we may argue that $\bar{V} \bar{\mu}$ represents a mean-field approximation for the maximum number of cells $c_{j}$ with $c_{j} \in N\left(c_{i}\right)$ that are affected by a defect in $c_{i}$ during one subsequent time step. Accordingly, we can define a function that maps a given $\bar{\mu}$ to the upper bound on the $\operatorname{MLE}\left(\lambda_{m}\right)$, i.e.

$$
\lambda_{m}(\bar{\mu})=\log (\bar{V} \bar{\mu})
$$

yields a mean-field approximation of $\lambda_{m}(\bar{\mu})$ if both $\bar{\mu}$ and $\bar{V}$ are known. We refer to the outcome of Eq. (8) as a mean-field approximation, since it is derived using the mean connectivity $\bar{V}$, hence, it makes of the discrepancies between $N\left|\left(c_{i}\right)\right|$, and it is only valid for $t \rightarrow \infty$. Clearly, the function given by Eq. (8) reaches its maximum for $\bar{\mu}=1$, which can occur if and only if all cells $c_{j}$ in $N\left(c_{i}\right)$ are affected by a defect present in $c_{i}$ at the $t$-th time step during one subsequent time step, and this holds for all $c_{i}$ in $\mathcal{T}^{*}$. Furthermore, since $\bar{\mu}$ is confined between zero and one, it is clear that the upper bound for the MLE drops as $\phi_{i}$ becomes less sensitive, expressed in terms of $\bar{\mu}$, to its input $\tilde{s}\left(N\left(c_{i}\right), t\right)$.

\section{Phenomenological study of irregular totalistic CA}

\subsection{Conventions}

Unless stated otherwise, the results presented in this section were obtained numerically for $T=500$, since by then both $\lambda$ and $\bar{\mu}$ showed convergence in the sense that an increase of $T$ did not significantly alter the numerically assessed values. Furthermore, periodic boundary conditions were applied in order to minimize boundary effects owing to the finiteness of $\mathcal{T}^{*}$ that, for the simulations considered in this section, consisted of 675 irregular cells covering a unit square, and were generated from random seeds in $[0,1]^{2}$ using a Voronoi tessellation. Using a Moore neighborhood, we obtained for this exemplary tessellation $\bar{V}=6.97$. As an exemplary family of irregular totalistic CA we consider in the remainder of this section 2-state, 5-sum irregular totalistic CA, for which $S=\{0,1\}$. Hence, in accordance with the enumeration scheme for irregular totalistic CA outlined in Section $264 \mathrm{CA}$ rules can be enumerated within this particular CA family.

\subsection{Phenomenology}

Figure 1 depicts the numerically evaluated $\operatorname{MLE}(\lambda)$ versus the geometric mean of the proportion of non-zero entries in $J(\bar{\mu})$ of the 2-state, $\theta=5$ irregular totalistic CA for which $\lambda \neq-\infty$, together with the function $\lambda_{m}(\bar{\mu})=\log (\bar{V} \bar{\mu})$, which, according to Eq. (8), for the tessellation used in these simulations equals $\lambda_{m}(\bar{\mu})=$ $\log (6.97 \bar{\mu})$. Besides, it displays a logarithmic function $\lambda_{m}(\bar{\mu})=\log \left(\overline{V^{*}} \bar{\mu}\right)$ that was fitted to $(\bar{\mu}, \lambda)$ data pairs. Since the $(\bar{\mu}, \lambda)$ enclosed within the demarcated area depicted in Fig. 1 clearly deviate from the overall trend that can be inferred from this figure, these data pairs were excluded from the fitting procedure. As 
such, we established $\overline{V^{*}}=6.77$ with a coefficient of determination $R^{2}=0.98$. The close agreement between the fitted function and the function given by Eq. (8) indicates the validity of the latter.

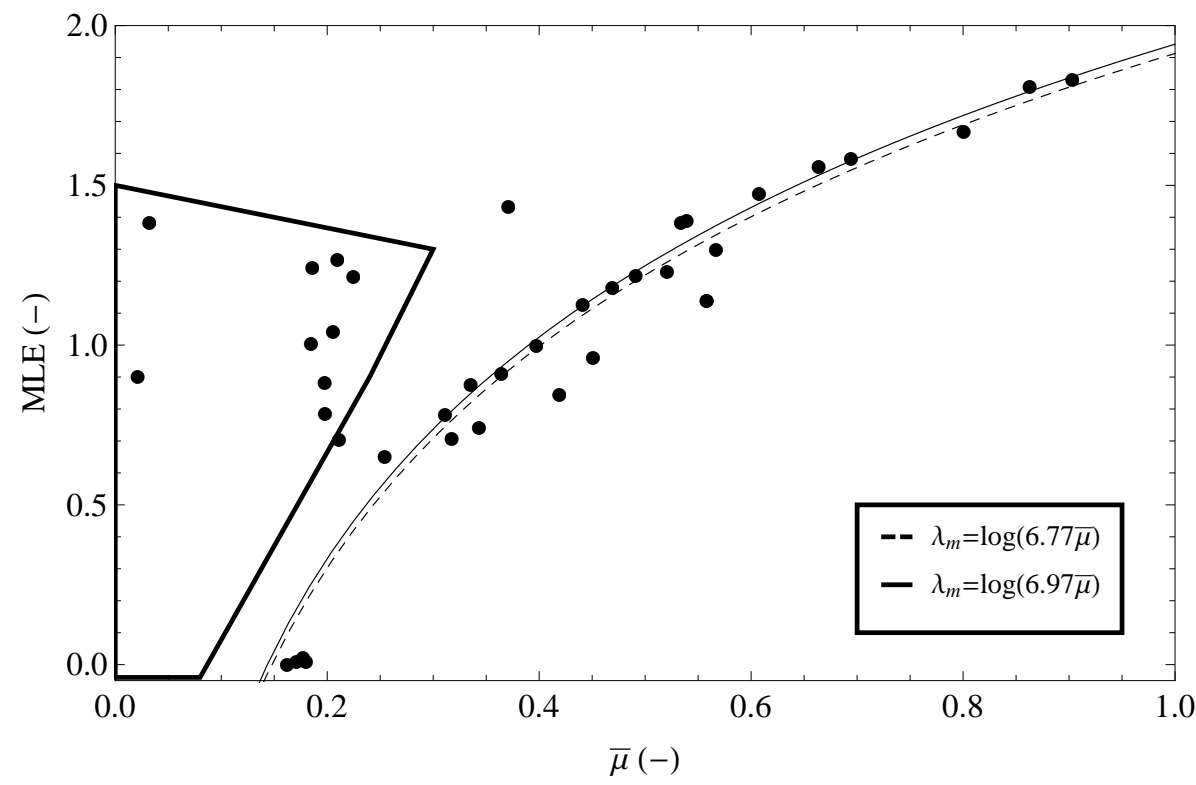

Fig. 1. Maximum Lyapunov exponent $(\lambda)$ versus the geometric mean of the proportion non-zero entries in $J(\bar{\mu})$ after 500 time steps, starting from a random initial condition. Results and are only shown for those 2-state, $\theta=5$ irregular totalistic CA for which $\lambda \neq-\infty$.

Though Figure 1 confirms the validity of Eq. (8), it also shows that the overall upper bound on the MLE, which is given by Eq. (8) for $\bar{\mu}=1$ such that $\lambda_{m}(\bar{\mu})=\log (6.97) \approx 1.94$, is not attained by any of the 64 considered 2-state, 5 -sum irregular totalistic CA. More specifically, the highest MLE, equaling 1.83, is found for rule 85 , which, at the same time gives rise to the highest $\bar{\mu}$ that is observed among the members of the CA family at stake, being $\bar{\mu}=0.9$. Hence, given the fact that none of the $\mathrm{CA}$ contained in the considered $\mathrm{CA}$ family attains the theoretical upper bound on $\bar{\mu}$, i.e. $\bar{\mu}=1$, meaning that there is no CA rule for which $J_{i j}=1$ for all $c_{i}$ in $\mathcal{T}^{*}$ and $c_{j} \in N\left(c_{i}\right)$, it is obvious that the overall upper bound on the MLE cannot be reached by any of the investigated CA rules. Yet, this finding gives inevitably gives rise to the question why none of the CA evolves towards $\bar{\mu}=1$. This issue can be elucidated by reconsidering the upper bound $\theta$ that was introduced in Section 2 to set up an enumeration scheme for $k$-state irregular totalistic CA. This upper bound $\theta$ entails all $\sigma_{i}$ for which $\sigma_{i} \geq \theta$ to be mapped to the same state, i.e. $\Omega\left(\sigma_{i}\right)=\Omega(\theta)$, and, as such, makes the totalistic CA partially insensitive to its input. For instance, for the 
CA family at stake, we chose $\theta=5$, such that $\Omega\left(\sigma_{i}\right)=\Omega(5)$ for all $\sigma_{i} \geq 5$. Yet, seen the exemplary tessellation has $\bar{V} \approx 7$ the existence of cells $c_{i} \in \mathcal{T}^{*}$ for which $\sigma_{i} \geq 5$ is certainly not unlikely, though this cannot be discerned by the CA since $\Omega\left(\sigma_{i}\right)=\Omega(5)$. Clearly, the lower the upper bound $\theta$ is chosen with respect to $\bar{V}$, the larger becomes its influence on the CA's dynamical properties.

Table 1, giving an overview of the $\lambda$ and $\bar{\mu}$ that were assessed numerically for the CA within the studied family of irregular totalistic CA, shows that 26 rules give rise to $\lambda=-\infty$ indicating that these $\mathrm{CA}$ evolve converging trajectories in phase space. For comprehensiveness, we must underline that, in the framework of this preliminary study, the values reported in Table 1 are obtained by considering only one perturbed initial configuration $s_{0}^{*}$ in order to curtail the amount of computation time, whereas a profound study of a CA's dynamical properties should be based upon an ensemble of perturbed initial configurations [3].

Table 1. Dynamical properties of 2 -state, $\theta=5$ irregular totalistic rules: sensitivity to initial conditions, expressed in terms of $\lambda$, and the sensitivity of $\Omega$ to its input $\sigma_{i}$, expressed by $\bar{\mu}$.

\begin{tabular}{c|cc|c|cc|c|cc|c|cc}
\hline \hline rule & $\bar{\mu}$ & $\lambda$ & rule & $\bar{\mu}$ & $\lambda$ & rule & $\bar{\mu}$ & $\lambda$ & rule & $\bar{\mu}$ & $\lambda$ \\
\hline 0 & 0 & $-\infty$ & 16 & 0 & $-\infty$ & 32 & 0 & $-\infty$ & 48 & $0.06-\infty$ \\
1 & 0.14 & $-\infty$ & 17 & 0.44 & 1.13 & 33 & 0.32 & 0.71 & 49 & $0.06-\infty$ \\
2 & 0.56 & 1.14 & 18 & 0.66 & 1.56 & 34 & 0.52 & 1.23 & 50 & $0.02-\infty$ \\
3 & 0.02 & $-\infty$ & 19 & 0.54 & 1.39 & 35 & 0.34 & 0.88 & 51 & $0.02-\infty$ \\
4 & 0.53 & 1.38 & 20 & 0.86 & 1.81 & 36 & 0.37 & 1.44 & 52 & $0.02-\infty$ \\
5 & 0.21 & 1.27 & 21 & 0.9 & 1.83 & 37 & 0.22 & 1.22 & 53 & $0.02-\infty$ \\
6 & 0.42 & 0.85 & 22 & 0.69 & 1.59 & 38 & 0.21 & 1.04 & 54 & $0.02-\infty$ \\
7 & 0.03 & $-\infty$ & 23 & 0 & $-\infty$ & 39 & 0.18 & 1.01 & 55 & $0.02-\infty$ \\
8 & 0.01 & $-\infty$ & 24 & 0.02 & 0.9 & 40 & 0.19 & 1.24 & 56 & 0 & $-\infty$ \\
9 & 0.61 & 1.47 & 25 & 0.47 & 1.18 & 41 & 0.2 & 0.89 & 57 & 0 & $-\infty$ \\
10 & 0.8 & 1.67 & 26 & 0.57 & 1.3 & 42 & 0.21 & 0.71 & 58 & 0 & $-\infty$ \\
11 & 0.03 & 1.39 & 27 & 0.49 & 1.22 & 43 & 0.2 & 0.79 & 59 & 0 & $-\infty$ \\
12 & 0.4 & 1. & 28 & 0.31 & 0.79 & 44 & 0.18 & 0.01 & 60 & 0 & $-\infty$ \\
13 & 0.45 & 0.96 & 29 & 0.36 & 0.91 & 45 & 0.18 & 0.02 & 61 & 0 & $-\infty$ \\
14 & 0.34 & 0.74 & 30 & 0.25 & 0.65 & 46 & 0.17 & 0.01 & 62 & 0 & $-\infty$ \\
15 & 0.07 & $-\infty$ & 31 & 0.08 & $-\infty$ & 47 & 0.16 & 0 & 63 & 0 & $-\infty$ \\
\hline \hline
\end{tabular}

\section{Discussion}

Despite the validity of Eq. (8) and the usability of Lyapunov exponents and Jacobian-based measures was demonstrated for the family of 2-state, 5 -sum totalistic CA, several issues concerning this approach are still awaiting closer inspection. First, the studied CA family encloses not more than 64 rules, whereas the usefulness of the approach should be checked against a much broader family. 
Second, the conclusions in this paper are drawn from one exemplary irregular tessellation, which makes it debatable whether part of the CA behavior observed is caused by the underlying tessellation, rather than by the CA's intrinsic properties. Third, and closely related to the second issue, concerns the effects on CA dynamics that may arise from using a regular rather than an irregular tessellation. In forthcoming work we hope to shed some light on each of these issues, and, by doing so, consolidating the approach discussed in this paper.

\section{Conclusions}

In this paper we proposed adequate measures, namely Lyapunov exponents and Jacobian-based measure, that are able to grasp the dynamics of a CA regardless the tessellation it is based upon, and to provide an objective means for comparing the dynamics of CA across different tessellations of $\mathbb{R}^{n}$. Further, the relationship between both allows to obtain a mean-field approximation of the upper bound on a CA's Lyapunov exponent. The soundness of both measures is illustrated by means of a simulation study in which we considered the family of 2-state, 5 -sum totalistic CA. In a forthcoming study, we employ both measures to quantitatively describe the dependence of a CA's dynamical properties on exploited tessellation.

Acknowledgments. The authors wish to acknowledge S. Wolfram and his coworkers for their commitment in organizing the yearly New Kind of Science Summer School, which served as a steppingstone for initiating this work.

\section{References}

1. Alexandridis, A., Vakalis, D., Siettos, C., Bafas, G.: A cellular automata model for forest fire spread prediction: The case of the wildfire that swept through Spetses Island in 1990. Appl. Math. Comp. 204, 191-201 (2008)

2. Baetens, J., De Baets, B.: Cellular automata on irregular tessellations. Chaos, Solitons Fractals (2010), submitted

3. Baetens, J., De Baets, B.: Phenomenological study of irregular cellular automata based on Lyapunov exponents and Jacobians. Chaos (2010), submitted

4. Bagnoli, F., Rechtman, R.: Synchronization and maximum Lyapunov exponents of cellular automata. Phys. Rev. E: Stat. Nonlinear Soft Matter Phys. 59, R1307R1310 (1999)

5. Bagnoli, F., Rechtman, R.: Thermodynamic entropy and chaos in a discrete hydrodynamical system. Phys. Rev. E: Stat. Nonlinear Soft Matter Phys. 79, 041115 (2009)

6. Bagnoli, F., Rechtman, R., Ruffo, S.: Damage spreading and Lyapunov exponents in cellular automata. Phys. Lett. A 172, 34-38 (1992)

7. Baltzer, H., Braun, P., Köhler, W.: Cellular automata models for vegetation dynamics. Ecol. Modell. 107, 113-125 (1998)

8. Batty, M.: Cellular dynamics: modelling urban growth as a spatial epidemic. In: Fischer, M., Leung, Y. (eds.) Geocomputational Modelling, pp. 109-141. Springer, Berlin, Germany (2001) 
9. Chopard, B., Luthi, P., Queloz, P.: Cellular automata model of car traffic in a two-dimensional street network. J. Phys. A: Math. Gen. 29, 2325-2336 (1996)

10. Coppola, E., Tomassetti, B., Mariotti, L., Verdecchia, M., Visconti, G.: Cellular automata algorithms for drainage network extraction and rainfall assimilation. Hydrol. Sci. J. 52, 579-592 (2007)

11. Courbage, M., Kamiński, B.: Space-time directional Lyapunov exponents for cellular automata. J. Stat. Phys. 124, 1499-1509 (2006)

12. Crisci, G., Iovine, G., Gregorio, S.D., Lupiano, V.: Lava-flow hazard on the SE flank of Mt. Etna (Southern Italy). J. Volcanol. Geotherm. Res. 177, 778-796 (2008)

13. D'Ambrosio, D., Spataro, W.: Parallel evolutionary modelling of geological processes. Parallel Comput. 33, 186-212 (2007)

14. Deutsch, A., Dormann, S.: Cellular Automaton Modeling of Biological Pattern Formation: Characterization, Applications, and Analysis. Birkhäuser, Bonn, Germany (2005)

15. Dewdney, A.: Sharks and fish wage an ecological war on the toroidal planet. Sci. Am. 251, 14-22 (1984)

16. Ilachinski, A.: Cellular Automata. A Discrete Universe. World Scientific, London, United Kingdom (2001)

17. Jackson, E.: Perspectives of Nonlinear Dynamics, vol. 1 and 2. Cambridge University Press, Cambridge, United Kingdom (1991)

18. Kier, L., Seybold, P., Cheng, C.: Modelling Chemical Systems using Cellular Automata. Springer, Dordrecht, The Netherlands (2005)

19. Langton, C.: Computation at the edge of chaos. Physica D 42, 12-37 (1990)

20. Mallet, D., De Pillis, L.: A cellular automata model of tumor-immune system interactions. J. Theor. Biol. 239, 334-350 (2006)

21. Milne, J., Fu, S.: Epidemic modelling using cellular automata. In: Proc. ACAL'03. pp. 43-57. Canberra (December 2003)

22. Parsons, J., Fonstad, M.: A cellular automata model of surface water flow. Hydrol. Processes 21, 2189-2195 (2007)

23. Picioreanu, C., van Loosdrecht, M., Heijnen, J.: Mathematical modeling of biofilm structure with a hybrid differential-discrete cellular automaton approach. Biotechnol. and Bioeng. 58, 101-116 (1998)

24. Pizarro, G., Griffeath, D., Noguera, D.: Quantitative cellular automaton model for biofilms. J. Environ. Eng. 127, 782-789 (2001)

25. Preziosi, L.: Cancer Modelling and Simulation. Chapman \& Hall, Boca Raton, United States (2003)

26. Shereshevsky, M.: Lyapunov exponents for one-dimensional cellular automata. J. Nonlinear Sci. 2, 1-8 (1991)

27. Tisseur, P.: Cellular automata and Lyapunov exponents. Nonlinearity 13, 1547$1560(2000)$

28. Urías, J., Rechtman, R., Enciso, A.: Sensitive dependence on initial conditions for cellular automata. Chaos 7, 688-693 (1997)

29. Valette, G., Prvost, S., Lucas, L., Lonard, J.: SoDA project: A simulation of soil surface degradation by rainfall. Computer Graphics 30, 494-506 (2006)

30. Vichniac, G.: Boolean derivatives on cellular automata. Physica D 45, 63-74 (1990)

31. von Neumann, J.: The general and logical theory of automata. In: Jeffress, L. (ed.) Cerebral Mechanisms in Behaviour: The Hixon Symposium, pp. 1-32. Wiley, New York, United States (1951)

32. von Neumann, J., Burks, A.: Theory of Self-Reproducing Automata. University of Illinois Press, Champaign, United States (1966) 
33. White, S., del Rey, A., Sanchez, G.: Modeling epidemics using cellular automata. Appl. Math. Comput. 186, 193-202 (2007)

34. Wolfram, S.: Algebraic properties of cellular automata. Commun. Math. Phys. 93, 219-258 (1984)

35. Wolfram, S.: Universality and complexity in cellular automata. Physica D 10, 1-35 (1984)

36. Wolfram, S.: Cellular Automata and Complexity. Westview Press, Boulder, United States (1994)

37. Wolfram, S.: A New Kind of Science. Wolfram Media Inc., Champaign, United States (2002)

38. Wuensche, A., Lesser, M.: The Global Dynamics of Cellular Automata, vol. 1. Addison-Wesley, London, United Kingdom (1992) 